University of Wollongong

Research Online

Faculty of Engineering and Information

Faculty of Engineering and Information

Sciences - Papers: Part A

Sciences

$1-1-2020$

\title{
High sensitivity face shear magneto-electric composite array for weak magnetic field sensing
}

Yun Lu

yl544@uowmail.edu.au

Zhenxiang Cheng

University of Wollongong, cheng@uow.edu.au

Jianguo Chen

Weihua Li

University of Wollongong, weihuali@uow.edu.au

Shujun Zhang

University of Wollongong, shujun@uow.edu.au

Follow this and additional works at: https://ro.uow.edu.au/eispapers

Part of the Engineering Commons, and the Science and Technology Studies Commons

Research Online is the open access institutional repository for the University of Wollongong. For further information contact the UOW Library: research-pubs@uow.edu.au 


\title{
High sensitivity face shear magneto-electric composite array for weak magnetic field sensing
}

\author{
Abstract \\ (C) 2020 Author(s). A magnetic field sensor is designed and fabricated using a piezoelectric face shear \\ mode $\mathrm{Pb}(\mathrm{Mg} 1 / 3 \mathrm{Nb} 2 / 3) 03-\mathrm{PbTiO3}$ (PMN-PT)/Metglas magneto-electric (ME) composite. An outstanding \\ ME coupling coefficient up to $1600 \mathrm{~V} /(\mathrm{cm} \mathrm{Oe})$ was experimentally achieved, being $\sim 50 \%$ higher than the \\ value from the extensional PMN-PT/Metglas ME composite with the same volume. The detection limit \\ was found to be $2 \times 10-6$ Oe for the DC magnetic field, while it was $2 \times 10-8$ Oe for the AC magnetic field. \\ The sensitivity of the face shear mode PMN-PT/Metglas ME composite is about one order of magnitude \\ higher than that of a 32 extensional mode PMN-PT/Metglas based ME composite in sensing a weak DC \\ magnetic field. A sensing array was also designed based on the ME composite to image weak DC \\ magnetic fields, demonstrating a great potential promising for sensing weak magnetic fields.

\section{Disciplines} \\ Engineering | Science and Technology Studies

\section{Publication Details} \\ Lu, Y., Cheng, Z., Chen, J., Li, W. \& Zhang, S. (2020). High sensitivity face shear magneto-electric \\ composite array for weak magnetic field sensing. Journal of Applied Physics, 128 (6),
}




\section{High sensitivity face shear magneto-electric composite array for weak magnetic field sensing}

Cite as: J. Appl. Phys. 128, 064102 (2020); https://doi.org/10.1063/5.0011931

Submitted: 28 April 2020 . Accepted: 28 July 2020 . Published Online: 11 August 2020

Yun Lu (D), Zhenxiang Cheng (D), Jianguo Chen (D), Weihua Li (D), and Shujun Zhang (D)

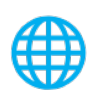

\section{ARTICLES YOU MAY BE INTERESTED IN}

Acceptor dopant mediated electrical property modification in $\mathrm{Bi}_{0.5} \mathrm{Na}_{0.5} \mathrm{TiO}_{3}$-based piezoceramic

Journal of Applied Physics 128, 064103 (2020); https://doi.org/10.1063/1.5144618

Defects chemistry in high-efficiency and stable perovskite solar cells

Journal of Applied Physics 128, 060903 (2020); https://doi.org/10.1063/5.0012384

Domain wall conductivity as the origin of enhanced domain wall dynamics in polycrystalline $\mathrm{BiFeO}_{3}$

Journal of Applied Physics 128, 064104 (2020); https://doi.org/10.1063/5.0017374

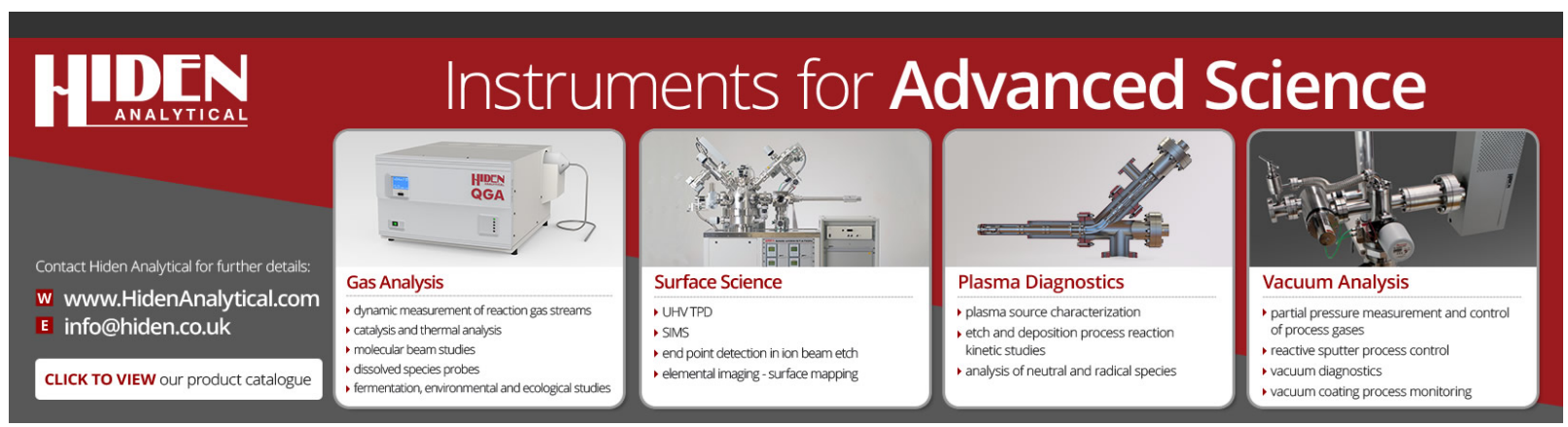




\title{
High sensitivity face shear magneto-electric composite array for weak magnetic field sensing
}

\author{
Cite as: J. Appl. Phys. 128, 064102 (2020); doi: 10.1063/5.0011931 \\ Submitted: 28 April 2020 - Accepted: 28 July 2020 . \\ Published Online: 11 August 2020
}

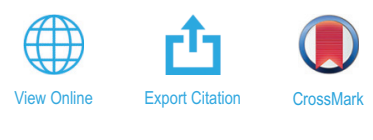

Yun Lu, ${ }^{1}$ (D) Zhenxiang Cheng, ${ }^{1}$ (D) Jianguo Chen, ${ }^{2}$ (D) Weihua Li, ${ }^{3}$ (iD and Shujun Zhang ${ }^{1, a)}$ (iD

AFFILIATIONS

${ }^{1}$ Institute for Superconducting and Electronic Materials, Australian Institute of Innovative Materials, University of Wollongong, Wollongong, NSW 2500, Australia

${ }^{2}$ School of Materials Science and Engineering, Shanghai University, Shanghai 200444, People's Republic of China

${ }^{3}$ School of Mechanical, Materials and Mechatronic Engineering, University of Wollongong, Wollongong, NSW 2522, Australia

${ }^{a)}$ Author to whom correspondence should be addressed: shujun@uow.edu.au

\begin{abstract}
A magnetic field sensor is designed and fabricated using a piezoelectric face shear mode $\mathrm{Pb}\left(\mathrm{Mg}_{1 / 3} \mathrm{Nb}_{2 / 3}\right) \mathrm{O}_{3}-\mathrm{PbTiO} 3(\mathrm{PMN}-\mathrm{PT}) / \mathrm{Metglas}$ magneto-electric (ME) composite. An outstanding ME coupling coefficient up to $1600 \mathrm{~V} /(\mathrm{cm} \mathrm{Oe}$ ) was experimentally achieved, being $~ 50 \%$ higher than the value from the extensional PMN-PT/Metglas ME composite with the same volume. The detection limit was found to be $2 \times 10^{-6}$ Oe for the DC magnetic field, while it was $2 \times 10^{-8}$ Oe for the AC magnetic field. The sensitivity of the face shear mode $\mathrm{PMN}-\mathrm{PT} /$ Metglas ME composite is about one order of magnitude higher than that of a 32 extensional mode PMN-PT/Metglas based ME composite in sensing a weak DC magnetic field. A sensing array was also designed based on the ME composite to image weak DC magnetic fields, demonstrating a great potential promising for sensing weak magnetic fields.
\end{abstract}

Published under license by AIP Publishing. https://doi.org/10.1063/5.0011931

\section{INTRODUCTION}

Magnetoelectric (ME) coupling devices have been actively studied for potential tools as weak magnetic field sensors, as alternatives to low-sensitivity Hall-base magnetometers, and expensive superconducting quantum interference devices (SQUIDs), for example, bio-magnetic sensing, ${ }^{1,2}$ where the sensitivity is a key challenge. Although SQUIDs can be used for weak magnetic field measurements, they require liquid helium cooling with obvious disadvantages in terms of cost, temperature isolation, and a considerable amount of energy during the operation.

Magnetic sensors in the form of composites with laminated piezomagnetic (PM)/piezoelectric (PE) layers have recently reported to possess many advantages due to their excellent magnetoelectric coupling characteristics, high sensitivity, and wide operating temperature range. ${ }^{3,4}$ In addition, the 2-2 layer-structured $\mathrm{ME}$ composite exhibits a low equivalent noise level of $\sim 1 \mathrm{pT} / \mathrm{Hz}^{1 / 2}$ in an AC magnetic field of $1 \mathrm{kHz} .^{5}$ The ability to sense a weak magnetic field is as low as $8 \times 10^{-6} \mathrm{Oe},{ }^{6}$ in stark contrast to the value of $3.5 \times 10^{-2}$ Oe achieved by a commercially available Hall effect magnetometer. ${ }^{7}$ Due to the low piezoelectric coefficient in many piezoelectric materials, however, the ME composite could only provide limited sensing resolution, which limited its ability to sense weak magnetic fields.

Compared to ferroelectric polycrystalline ceramics, the $\mathrm{Pb}$ $\left(\mathrm{Mg}_{1 / 3} \mathrm{Nb}_{2 / 3}\right) \mathrm{O}_{3}-\mathrm{PbTiO}_{3}$ (PMN-PT) relaxor ferroelectric single crystal provides outstanding $\mathrm{PE}$ characteristics [piezoelectric charge coefficient, $d_{32} \approx-1200 \mathrm{pC} / \mathrm{N}$ vs $d_{31} \approx 300 \mathrm{pC} / \mathrm{N}$ in lead zirconate titanate (PZT) ceramics]., The ME composite based on mechanically coupled PMN-PT and Metglas shows a remarkable enhancement in its capability to convert a magnetic field into electric signals. $^{10}$ A longitudinally magnetized, transversely poled (L-T) PMN-PT/Metglas composite was found to have a ME coupling coefficient as large as $1100 \mathrm{~V} /(\mathrm{cm} \mathrm{Oe})$ at its resonant frequency. ${ }^{11}$ The highest piezoelectric charge coefficient of $3000 \mathrm{pC} / \mathrm{N}$ has been reported in the thickness shear mode of PMN-PT; ${ }^{12}$ however, its low mechanical quality factor $\left(Q_{m} \text { of } 20-30\right)^{13}$ will lead to a high mechanical loss, ${ }^{14}$ which greatly limits its ME coupling performance.

It is interesting to note that the face shear mode can be achieved in a $\mathrm{Zt} \pm 45^{\circ}$ cut [011] oriented PMN-PT single crystal, possessing high piezoelectric charge coefficients ( $d_{36}$ of up to $1700 \mathrm{pC} / \mathrm{N})^{15}$ and a good mechanical quality factor $\left(Q_{m} \geq 120\right){ }^{16}$ thus attracting extensive attentions for actuator, ${ }^{17}$ sensor, ${ }^{18}$ and 
motor applications. ${ }^{19-21}$ Recently, an ME sensor based on a face shear mode $\mathrm{Pb}\left(\mathrm{Mg}_{1 / 3} \mathrm{Nb}_{2 / 3}\right) \mathrm{O}_{3}-\mathrm{Pb}(\mathrm{ZrTi}) \mathrm{O}_{3}$ (PMN-PZT)/Metglas composite sensor was recently reported to possess outstanding sensitivity to AC magnetic fields. ${ }^{22}$ However, the potential sensing capability of the face shear mode ME sensor to both AC and DC magnetic fields needs further comprehensive investigation, where the anisotropic characteristic and its application have not been reported.

In this research, we systematically study the property of the face shear PMN-PT/Metglas ME composite. The sensitivity and ME coupling property were theoretically calculated and experimentally confirmed. Then, an ME sensor array was designed and fabricated using the proposed face shear mode PMN-PT/Metglas ME composite. The ME composite array is able to determine the accurate spatial location of a weak static magnetic field, by using the anisotropic property of the ME composite. This work suggests great potential of our piezoelectric ME composite array design for sensing of weak magnetic signals, in both DC and AC magnetic fields.

\section{EXPERIMENTAL SETUP}

To prepare the face shear ME composite, the PMN-PT single crystal sample was prepared by rotating at a $45^{\circ}$ angle along the $z$ axis [011] direction, cutting into a square-shaped plate with dimensions of $10 \times 10 \times 0.5 \mathrm{~mm}^{3}$, as shown in Fig. 1(a). The specifically designed dimensions were to ensure that the crystal could operate in the 36 -face shear mode to utilize its outstanding piezoelectric properties. ${ }^{15}$ Silver electrodes were deposited on the two [011] surfaces, and then the crystal was poled at the electric field of $10 \mathrm{kV} / \mathrm{cm}$ at room temperature. The poled PMN-PT single crystal was bound with Metglas foil (2605SA1) using an electrically conducting epoxy (MG Chemicals silver conductive epoxy adhesive 8330) and then cured for $24 \mathrm{~h}$ at room temperature. The impedance spectrum of the fabricated ME composite was characterized using an impedance analyzer (HP4294A, Agilent Technologies Inc.). The ME composite, shield, and solenoid coil were integrated into the sensing element as shown in Fig. 1(b). The ME composite was placed in the nonmagnetic non-conducting shield to isolate it from the electric noise of the environment. A solenoid coil was employed to generate an AC magnetic field. When the AC magnetic field alternately changes along the diagonal direction of the composite, the Metglas foil will generate in-plane displacement in the PMN-PT crystal to move along the AC magnetic field, leading to in-plane displacement, from which electric charge will be induced on the two surfaces of the PMN-PT crystal based on the piezoelectric effect. ${ }^{23,24}$ The output AC voltage signal is measured by a lock-in amplifier (SR 830, Stanford Research).

\section{RESULTS AND DISCUSSION}

\section{A. Operation mode and the calculated equivalent magnetic noise density (EMND)}

Figure 1(c) shows a measured impedance spectrum of the ME composite operating in the free vibration mode over the frequency range of $40 \mathrm{~Hz}-120 \mathrm{kHz}$ at room temperature. The resonant frequency and anti-resonant frequency were found to be $85 \mathrm{kHz}$ and $107 \mathrm{kHz}$, respectively. For a rectangular shaped composite, the resonant frequency can be evaluated by ${ }^{15}$

$$
f_{r}=\frac{F}{a+b} \sqrt{\frac{1}{\bar{\rho} \cdot \vec{s}}}
$$

where $F \sim 1.28$ is a correction constant, $a$ is the length and $b$ the width of the composite, $\bar{\rho} \sim 8 \mathrm{~g} / \mathrm{cm}^{3}$ is the equivalent density of the composite, and $\bar{s} \sim 65 \mathrm{pm}^{2} / \mathrm{N}$ is the equivalent elastic compliance of the composite, which can be estimated by ${ }^{25}$

$$
\frac{1}{\bar{s}}=\left[\frac{n_{c}}{s_{c}}+\frac{n_{l}}{s_{l}}\right],
$$

where $n_{c}$ and $n_{l}$ are the volume ratios of the crystal and load components (epoxy and Metglas), respectively, and $s_{c}$ and $s_{l}$ are the elastic compliance values of the crystal and load components (epoxy and Metglas), respectively. For the proposed design, the calculated resonant frequency is $87 \mathrm{kHz}$, which is very close to the measured value of $85 \mathrm{kHz}$. The minor difference is attributed to the composite fabrication process and the error in calculating the equivalent parameters. The dielectric loss tangents $\tan (\delta)$ is found to be $\sim 1.6 \%$ due to the epoxy adhesive, while the mechanical quality factor of the composite can be evaluated by $Q=f_{r} / \Delta f$, which is found to be $\sim 110$. The details of parameters of the composite are given in Table I.

The theoretical sensitivity of the fabricated sensor can be evaluated by determination of the equivalent magnetic noise density (EMND), ${ }^{26}$ which consists of the dielectric loss noise and the impedance thermal noise, and it can be calculated from the following equation: ${ }^{27,28}$

$$
\mathrm{EMND}=\frac{\sqrt{\frac{4 \cdot k \cdot T \cdot C_{p} \cdot \tan \delta}{2 \cdot \pi \cdot f}}+\sqrt{\frac{4 \cdot k \cdot T}{(2 \cdot \pi \cdot f)^{2} \cdot Z^{\prime}}}}{t_{p} \cdot \alpha_{M E}},
$$

where $\sqrt{\frac{4 \cdot k \cdot T \cdot C_{p} \cdot \tan \delta}{2 \cdot \pi \cdot f}}$ is the dielectric loss noise, $\sqrt{\frac{4 \cdot k \cdot T}{(2 \cdot \pi \cdot f)^{2} \cdot Z^{\prime}}}$ is the impedance thermal noise, and $t_{p}$ and $\alpha_{M E}$ represent the thickness of the crystal and the ME coupling coefficient, respectively. For the noise due to the dielectric loss $\sqrt{\frac{4 \cdot k \cdot T \cdot C_{p} \cdot \tan \delta}{2 \cdot \pi \cdot f}}$, where $k, T, C_{p}, \tan (\delta)$, and $f$ represent Boltzmann's constant, the temperature in kelvin, the capacitance of the composite, the dielectric loss, and the frequency, respectively. For the impedance thermal noise $\sqrt{\frac{4 \cdot k \cdot T}{(2 \cdot \pi \cdot)^{2} \cdot Z^{\prime}}}, Z^{\prime}$ is the real part of the impedance. EMND was calculated based on the measured impedance as function of frequency at room temperature, where the measurement was performed in the grounded noise isolation box to avoid external electromagnetic noise. Figure 1(d) displays the calculated EMND curve of the ME composite. The corresponding magnetic noise density is found to be $0.1 \mathrm{pT} / \mathrm{Hz}^{1 / 2}$ at the antiresonant frequency of $107 \mathrm{kHz}$, which is much improved compared to the resolution of the reported $\mathrm{ME}$ magnetic field sensor $\left(0.5 \mathrm{pT} / \mathrm{Hz}^{1 / 2}\right) .^{29}$ The low EMND allows the fabricated ME sensor to give a clear signal at a weak magnetic field, contributing to the improved sensitivity of the device. 
(a)

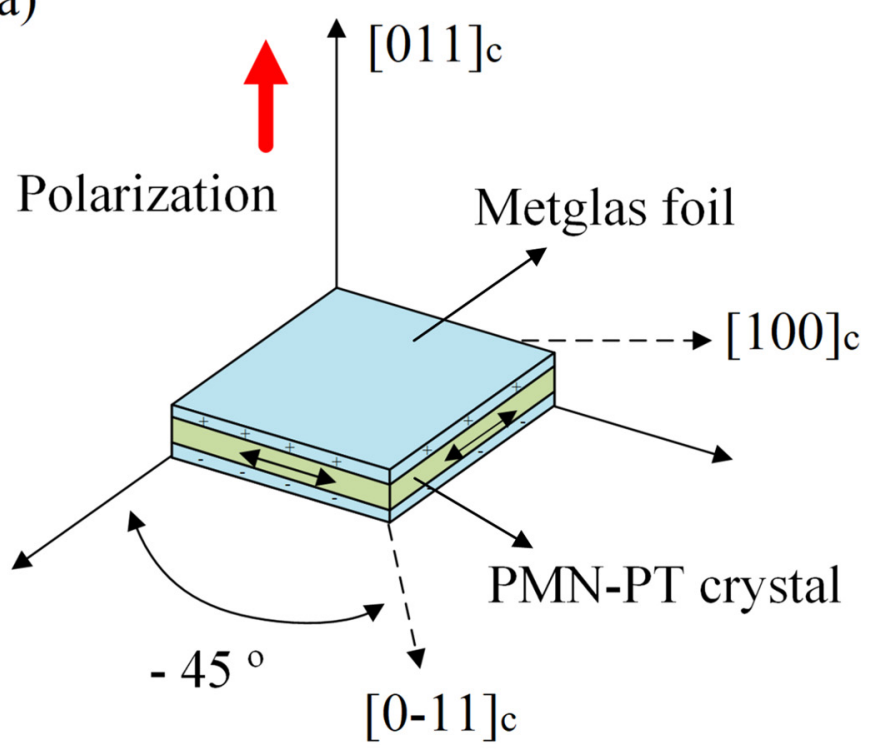

(b) (c)

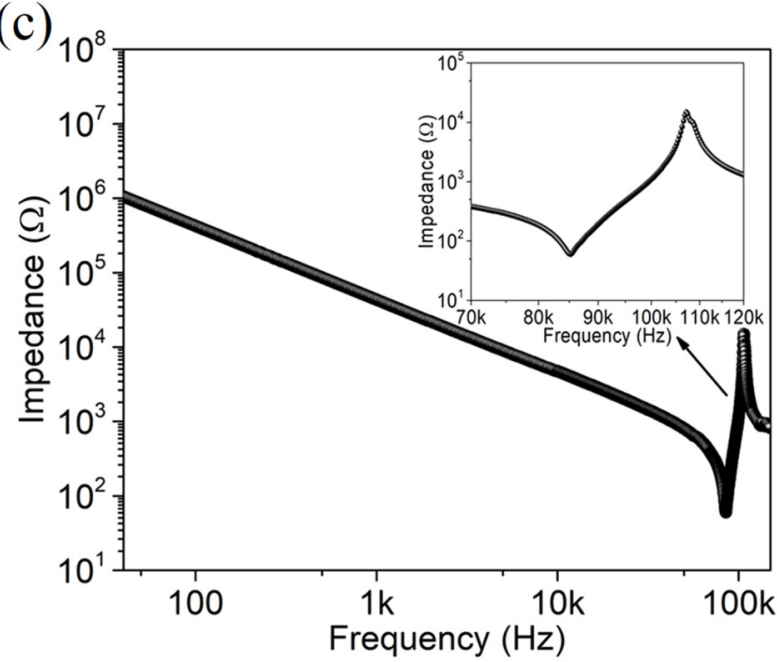

(d)
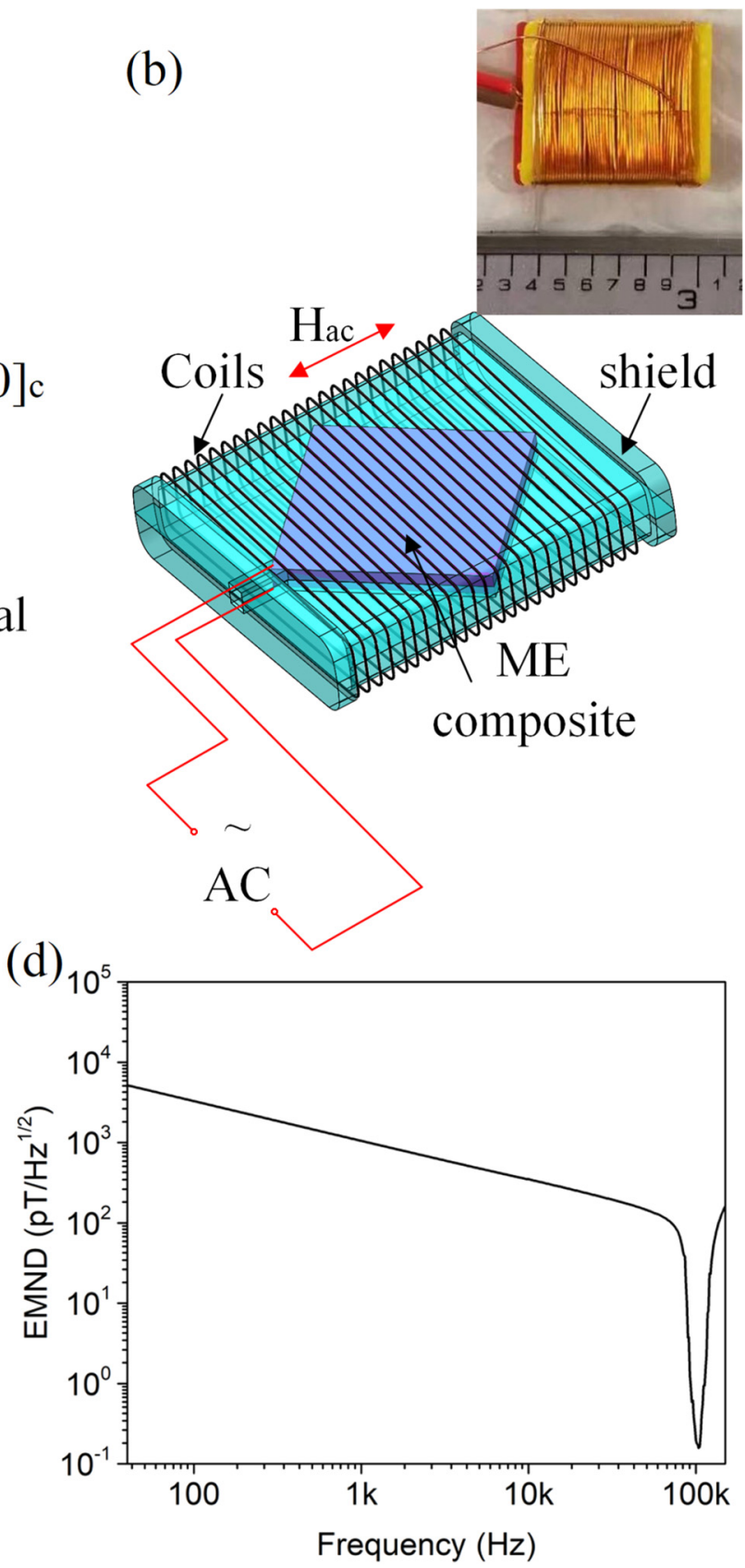

FIG. 1. (a) Schematic view and operation principle of the face shear mode ME composite. (b) Schematic view and photograph of the sensing element. (c) Impedance spectrum of the face shear PMN-PT/Metglas composite, with enlargement in the inset. (d) Calculated equivalent magnetic noise density (EMND) of the magnetic field sensor.

\section{B. ME coupling properties of the face shear mode ME composite}

The ME coupling property at the off resonant condition is tested at a quasi-static frequency of $1 \mathrm{kHz}$. The magnetic field is generated by Helmholtz coils. The detailed parameter of the
Helmholtz coils is listed in Table II. Figure 2(a) shows that the room temperature $\mathrm{ME}$ response depends on the DC magnetic field with the AC magnetic field fixed at $H_{a c}$ of $0.1 \mathrm{Oe}(1 \mathrm{kHz})$. The output $\mathrm{AC}$ voltage shows a near-linear increase with an increasing DC bias magnetic field up to 6 Oe. The maximum output voltage 
TABLE I. Parameter details of the face shear mode PMN-PT/Metglas ME composite.

\begin{tabular}{ccccccccc}
\hline \hline Parameters & Length $(\mathrm{mm})$ & Width $(\mathrm{mm})$ & Thickness $(\mathrm{mm})$ & $f_{r}(\mathrm{~Hz})$ & $f_{a}(\mathrm{~Hz})$ & $d_{36}(\mathrm{pC} / \mathrm{N})$ & $\tan (\delta)(\%)$ & $Q$ \\
\hline \hline & 10 & 0.61 & 85220 & 107160 & 1700 & 1.6 & 110 \\
\hline \hline
\end{tabular}

of $\sim 30 \mathrm{mV}(1 \mathrm{kHz})$ was acquired at $H_{d c}$ of 6 Oe. The equivalent ME coupling coefficient was calculated by $\alpha_{M E}=\frac{\partial E}{\partial H}$, ${ }^{23,30-33}$ leading to the maximum quasi-static $\alpha_{M E}$ being on the order of $\sim 6 \mathrm{~V} /(\mathrm{cm} \mathrm{Oe})$. From this experiment, it is obvious that the intensity of the DC magnetic field can be measured by the generated voltage in the $\mathrm{ME}$ composite. The ME coupling coefficient tends to decrease at higher magnetic field, due to the magnetostriction of the Metglas as it approaches saturation. ${ }^{34}$ The inset gives the first derivative of the $\alpha_{M E}$, which is calculated by $D_{M E}=\Delta \alpha_{M E} / \Delta H$. Note that the maximum $D_{M E}$ and the maximum voltage are not simultaneously achieved at the same magnetic field. In this case, the maximum $D_{M E}$ is acquired at the DC magnetic field of $H_{d c}=2.2$ Oe. This can be attributed to the increase of magnetic saturation in the Metglas foil under a DC magnetic field larger than 2.2 Oe.

The dynamic ME coefficient was measured as a function of excitation frequency under the fixed DC bias magnetic field, $H_{d c}=6$ Oe. As shown in Fig. 2(b), a giant ME coupling coefficient calculated based on $\alpha_{M E}=\frac{\partial E}{\partial H}$ was found to be $\sim 1600 \mathrm{~V} /(\mathrm{cm} \mathrm{Oe})$ at the anti-resonant frequency of $107.6 \mathrm{kHz}$, which is $\sim 50 \%$ higher than that reported for a 32 extensional mode PMN-PT based ME composite with the same volume. ${ }^{11}$ The enhancement of the ME coupling coefficient is attributed to the improved piezoelectric charge coefficient $\left(d_{36} \sim 1700 \mathrm{pC} / \mathrm{N}\right.$ vs $\left.d_{32} \sim 1200 \mathrm{pC} / \mathrm{N}\right)$ and mechanical quality factor for the face shear mode.

\section{Anisotropic characteristic of the ME composite and the magnetic sensitivity}

In order to determine the sensitivity of the ME sensor, the voltage output of the $10 \times 10 \times 0.5 \mathrm{~mm}^{3}$ face shear PMN-PT/ Metglas ME composite was measured under different amplitudes of $\mathrm{AC}$ and DC magnetic fields at its anti-resonant frequency. Figure 3(a) displays the measured AC voltage as a function of $H_{a c}$ with a DC magnetic field $H_{d c}=6$ Oe. Note that the magnetic field sensor exhibits a clear ME response to a weak AC magnetic field as low as $2 \times 10^{-8} \mathrm{Oe}$ at an anti-resonant frequency of $107.6 \mathrm{kHz}$ with the ME voltage output being on the order of $\sim 4.5 \mu \mathrm{V}$. The detection limit $2 \times 10^{-8}$ Oe of the studied sensor is much improved than the reported ME composite sensor $\left(9.2 \times 10^{-8} \mathrm{Oe}\right){ }^{35}$ The voltage output increases linearly as the AC magnetic field increases with a slope of $\sim 80 \mathrm{~V} / \mathrm{Oe}$. The calculated ME coupling coefficient is found to be $\sim 1600 \mathrm{~V} /(\mathrm{cm} \mathrm{Oe})$, which is in a good agreement with the measured dynamic ME coupling coefficient. Figure 3(b)

TABLE II. Parameter of the Helmholtz coils.

\begin{tabular}{ccccc}
\hline \hline Parameter & Diameter $(\mathrm{mm})$ & Turns & $\operatorname{Max} I(\mathrm{~A})$ & Resistance $(\Omega)$ \\
\hline 70 & 30 & 3 & 5 \\
\hline \hline
\end{tabular}

presents the ME voltage as a function of time in a step-change $H_{a c}$ from 0 (noise) to $2 \times 10^{-7} \mathrm{Oe}$ at frequency of $107.6 \mathrm{kHz}$. A noise of $\sim 2 \mu \mathrm{V}$ was measured at the frequency of $107.6 \mathrm{kHz}$. This noise is attributed to the combination of the impedance noise, thermal noise from the ME composite, and the noise from the lock-in amplifier and electric circuit. The experimental result shows a good repeatable output in response to the AC magnetic field. All these results demonstrate that the studied magnetic field sensor possesses a high sensitivity to weak AC magnetic fields with high reliability. Furthermore, the feasibility of calibrating a weak AC magnetic field via the output $\mathrm{AC}$ voltage of the sensor can be experimentally proved.

Figure 3(c) shows the maximum voltage output vs the angle between the $x$ and $y$ directions at $H_{d c}=6$ Oe. The maximum output is found to be about $30 \mathrm{mV}$ when the rotation angle reached $50^{\circ}$ and $225^{\circ}$, respectively. Meanwhile, the minimum voltage is found to be $\sim 8 \mathrm{mV}$ at $120^{\circ}$ and $315^{\circ}$, respectively, showing a strong anisotropic characteristic. This result agrees with the anisotropic behavior of the face shear piezoelectric constant $d_{36}$ of the PMN-PT crystal. $^{36}$ Therefore, the relative position between the DC magnetic target and the sensor can be monitored by measuring the output AC voltage. Figure $3(\mathrm{~d})$ displays the ME coupling property under a weak DC magnetic field. The detection limit is about $2 \times 10^{-6} \mathrm{Oe}$, which is much improved over that reported for an ultra-sensitive ME sensor, being on the order of $8 \times 10^{-6}$ Oe. $^{6}$ Clearly, the calculated response of the ME sensor to a weak $H_{d c}$ field is found to be $\sim 1 \mathrm{~V} / \mathrm{Oe}$ at $H_{d c} \leq 1 \times 10^{-4} \mathrm{Oe}$, being decreased to $\sim 5 \mathrm{mV} / \mathrm{Oe}$ for $H_{d c}=6$ Oe due to the magnetization saturation of Metglas at elevated $H_{d c}$.

\section{Magnet target sensing using the sensing array}

Sensing the position of a magnet target is one of the applications of the ME composite. ${ }^{37-40}$ To achieve the accurate position of the magnetic field source, one of the challenges is to isolate unwanted noise from the original output AC signal. To solve this problem, a sensing array was developed based on the ME composite. To build the sensing array, a total 43 pieces of the face shear mode PMN-PT/Metglas ME sensing elements were arrayed in a line as shown in Fig. 4(a). A permanent magnet with dimensions of $10 \times 10 \times 30 \mathrm{~mm}^{3}$ was used as a target. Figure 4 (b) displays the simulated distribution of the magnetic flux around the magnet and the sensor array. Because of the relative positions of the array and the magnet, the angle between the magnetic flux from the magnet and each sensing element is different. A lock-in amplifier (SR 830) was used to measure the output voltage from each sensing element and then the output results were built into a $1 \mathrm{D}$ voltage curve. The small difference in the angle is reflected in the output voltage curve of the array due to the anisotropic nature of the device. By processing the voltage curve, the contribution of the magnetic field 
(a)

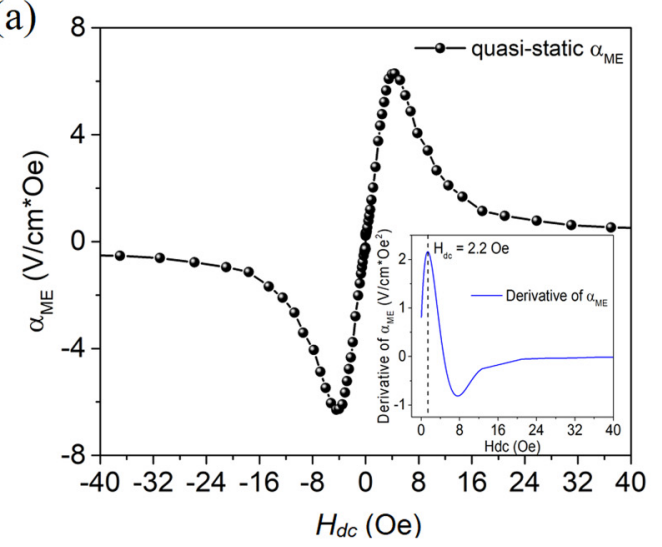

(b)

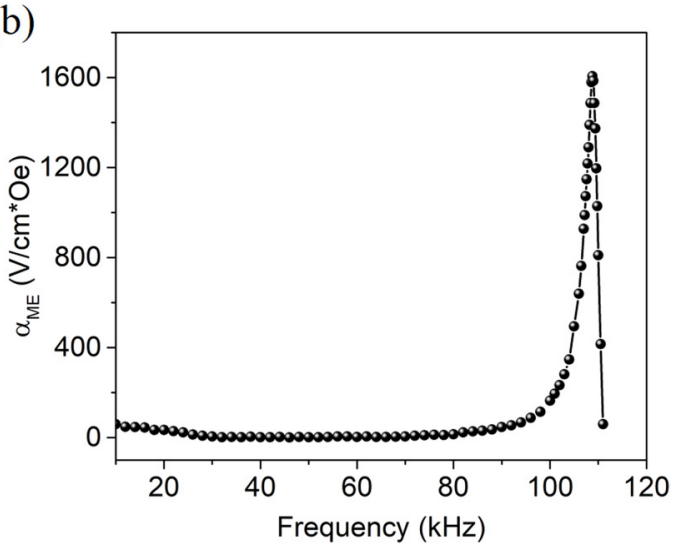

FIG. 2. ME coupling property of the ME composite sensor. (a) Measured quasi-static direct ME coefficient as a function of the magnetic bias $H_{d c}$ at the AC magnetic field $H_{a c}=0.10 \mathrm{e}(1 \mathrm{kHz})$, while the first derivative of $\alpha_{M E}$ is given in the inset. (b) Measured ME coupling coefficient as a function of frequency at the optimal DC magnetic field of 6 Oe.

(a)

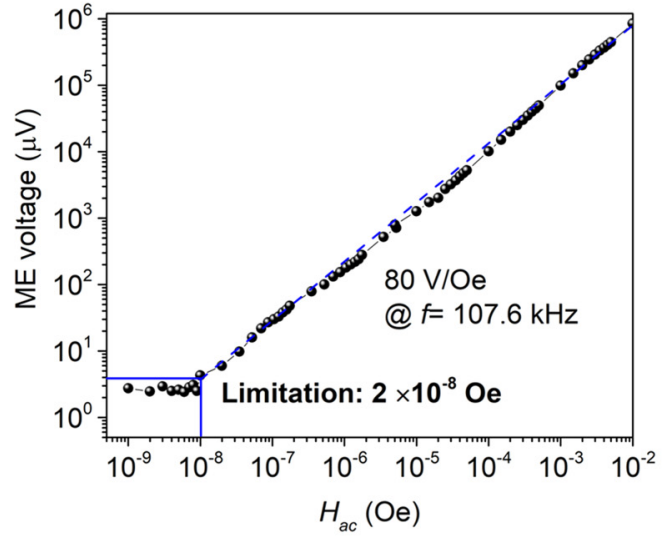

(c) $H_{d c}=6$ Oe, measured @ $@ f_{a}$

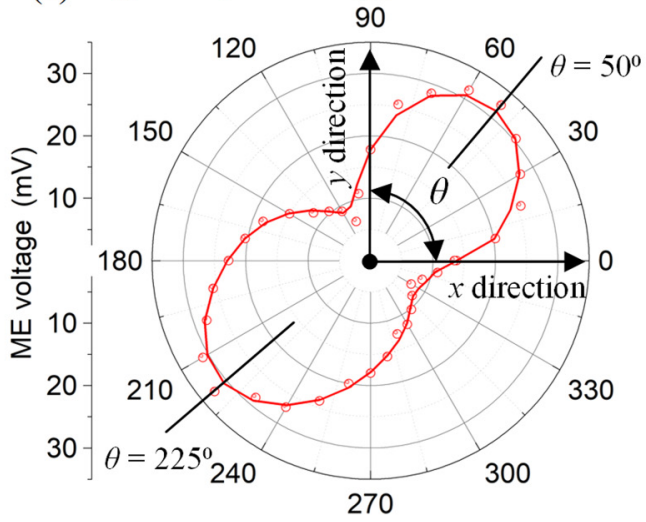

(b)

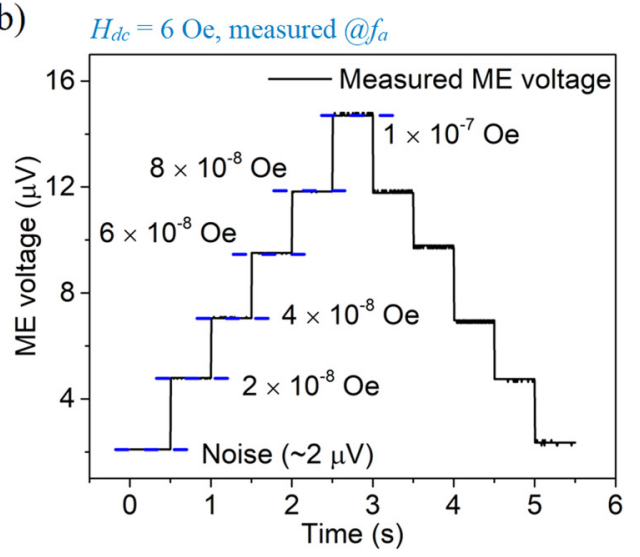

(d)

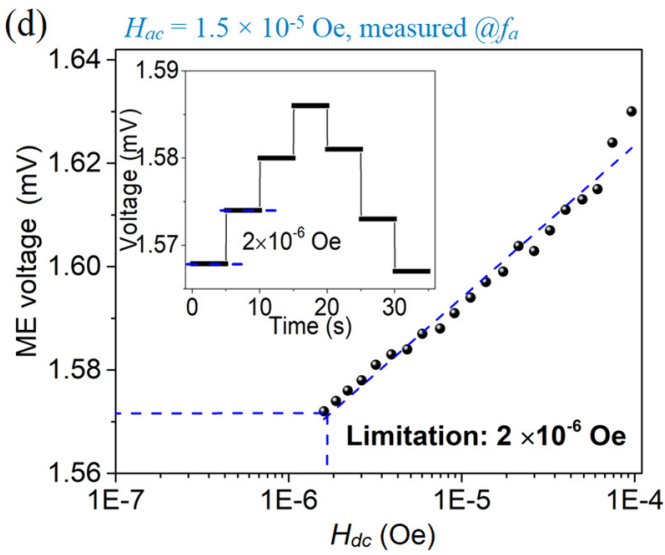

FIG. 3. AC magnetic field sensing. (a) Determination of the detection limit for the $A C$ magnetic field and ME voltage output of the magnetic field sensor as a function of $\mathrm{AC}$ magnetic fields. (b) Step-change measurement of the magnetic sensitivity to a small range of $\mathrm{AC}$ magnetic field from $2 \times 10^{-8} \mathrm{Oe}$ to $1 \times 10^{-7} \mathrm{Oe}$. (c) The ME response depends on the angle between the $x$ direction (i.e., [0-11] direction of the crystal) and the $y$ direction (direction of the DC magnetic field). (d) The detection limit for the DC magnetic field, with a step-change test of the output voltage as a function of time under different DC magnetic fields given in the inset. 
(a)

\section{magnet}
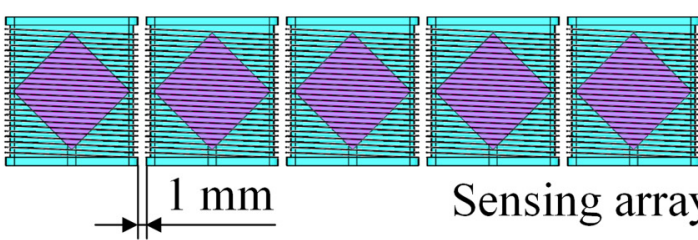

Sensing array (b)
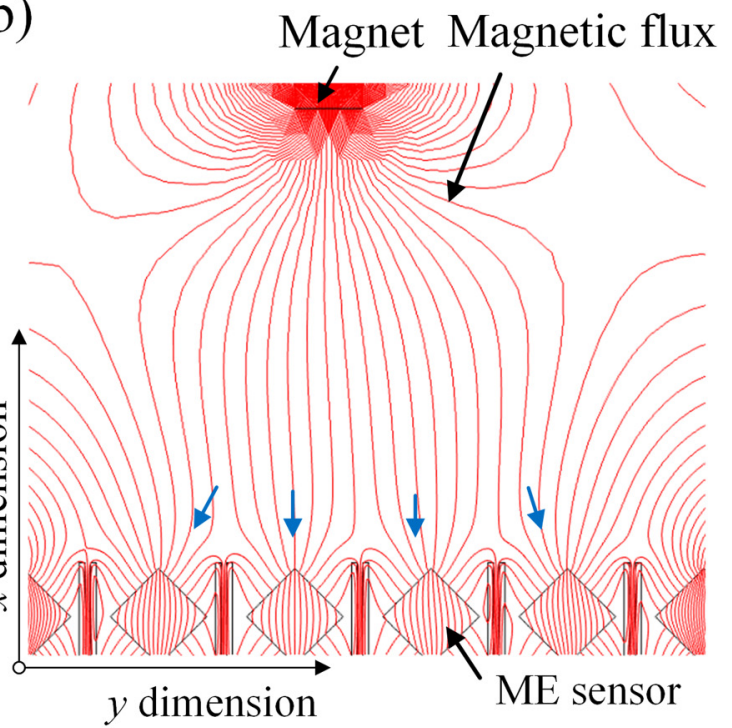

(c)

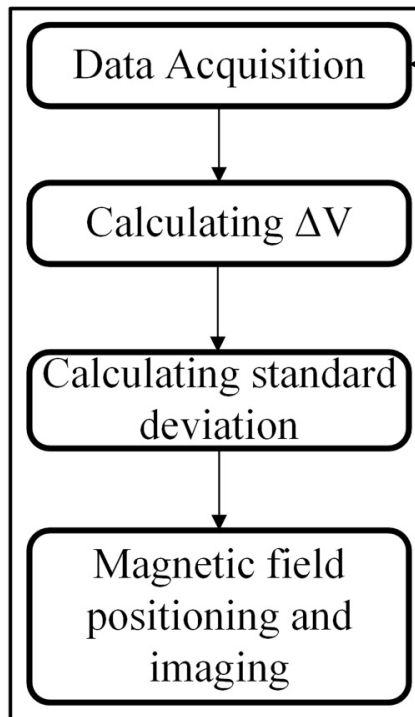

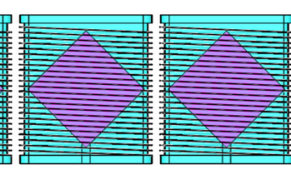

(1)
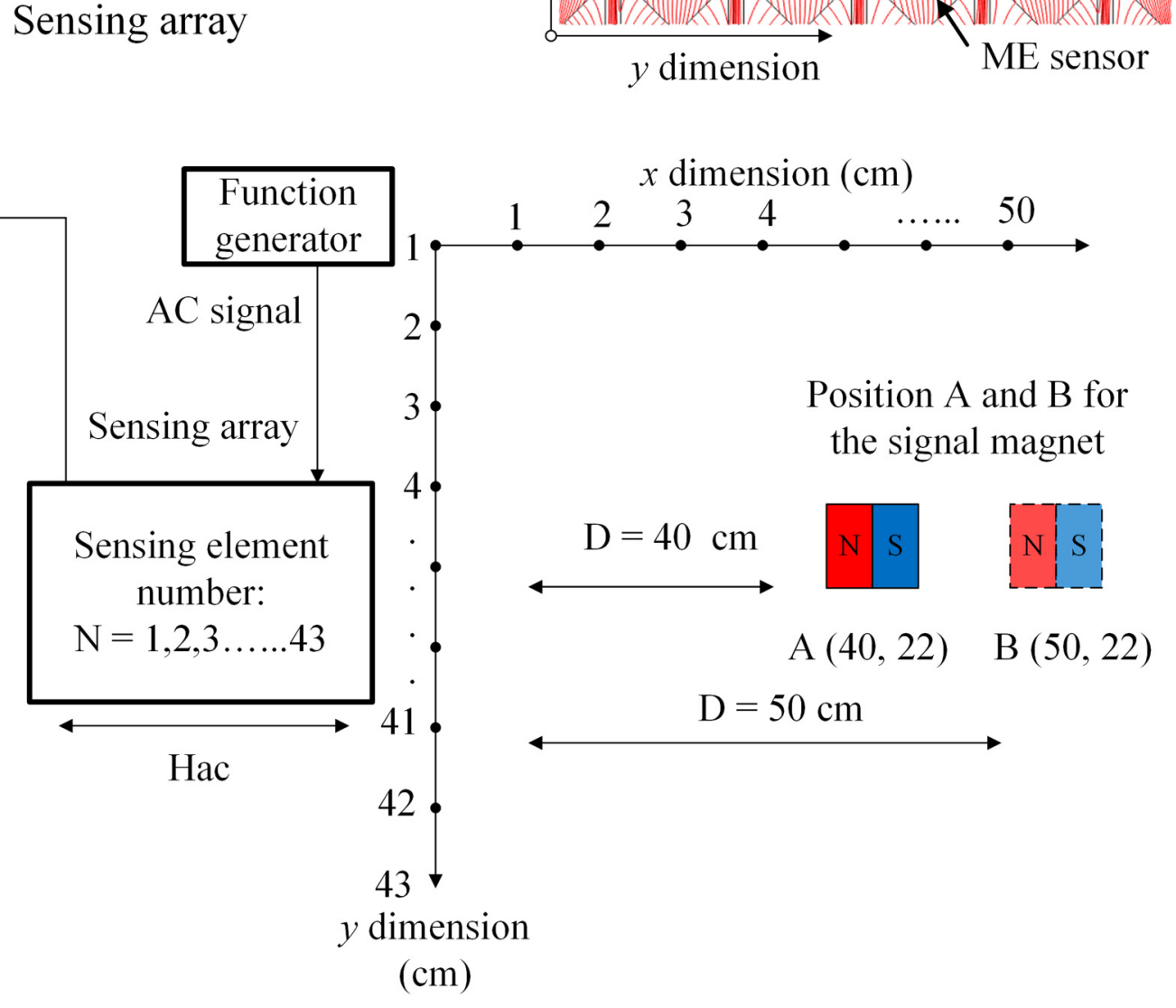

FIG. 4. (a) Schematic view of the sensing array. (b) Simulated magnetic flux distribution in the sensing array. (c) The process of magnetic field positioning and imaging.

intensity can be isolated, and the real position of the magnet target can be confirmed.

To generate a weak magnetic field, the target magnet was placed at the $A(40,22)$ and $B(50,22)$ positions, as shown in Fig. 4(c). The numbers 40 and 22 of the $(40,22)$ coordinate represent the distances between the sensing array and the magnet target along the $x$ axis and $y$ axis in centimeters, respectively. By using the finite element analysis model, the magnetic field was found to be on the order of $10^{-5}$ Oe and $10^{-6}$ Oe at the $\mathrm{A}$ and $\mathrm{B}$ positions, respectively. The difference in output voltage between the neighboring sensing 

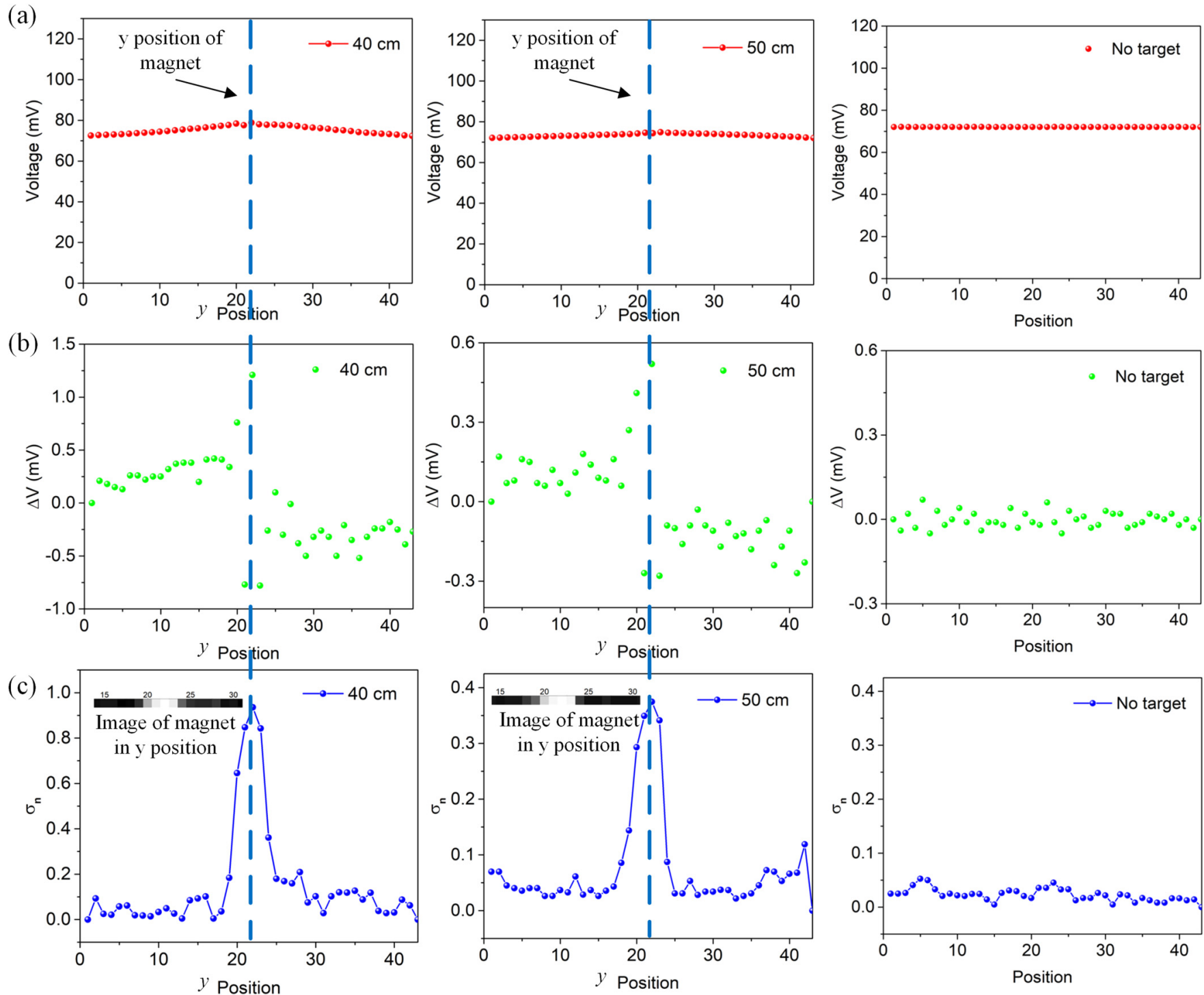

FIG. 5. (a) Measured output voltage from the sensing array when the magnet is placed at the $(40,22)$ and $(50,22)$ positions, compared to the scenario without a magnet target. (b) Calculated increment of the output voltage. (c) Calculated standard deviation of the signal and the image of the magnet (inset).

elements was calculated by $\Delta V_{n}=V_{n}-V_{n-1}$. Then, the standard deviation of $\Delta V$ can be calculated by

$$
\sigma_{n}=\sqrt{D_{n}}=\sqrt{E\left[\left(x_{n}-\bar{x}\right)^{2}\right]}
$$

where $x_{n}$ is the $\Delta V$ at position $n$ and $\bar{x}$ is the mean value of $\Delta V$, so the position of magnet target can be detected by confirming the maximum $\sigma_{n}$. The advantage of using maximum $\sigma_{n}$ is that the intensity of the detected magnetic field has a minimal effect on the exact position of the magnet target.
Figure 5(a) displays the measured output voltage curve when the magnet is placed at the $A(40,22)$ and $B(50,22)$ positions, and comparing the scenario without a magnet target. Note that there is no change in the output voltage curve when the magnet target is absent in the sensing area. Some minor fluctuation in $\Delta V$ and $\sigma_{n}$ can be neglected. However, a clear change in the ME output voltage from the sensors can be observed when the magnet source is placed in the sensing area. The output voltage curve indicates that the ME coupling output of the sensing array is impacted by both the magnetic field strength and the position of the magnet target. For the magnet at position A, the amplitude of the center 
voltage is $\sim 79 \mathrm{mV}$, while the peak output voltage is $\sim 75 \mathrm{mV}$ for position $\mathrm{B}$. The difference between the two peak voltages is attributed to the difference in the weak DC magnetic field intensity being detected by the sensing array. The curves in Fig. 5(b) present the calculated $\Delta V$, where the maximum $\Delta V$ is on the order of $\sim 1.2 \mathrm{mV}$ and $0.6 \mathrm{mV}$ for the magnet located at positions $\mathrm{A}$ and $\mathrm{B}$, respectively. $\Delta V$ consists of the contributions from the intensity of the magnetic field and the relative position between the sensor array and the magnet target. To acquire the accurate position of the magnet, the standard deviation of $\Delta V$ is calculated, as given in Fig. 5(c). As expected, the peak value of the standard deviation reflects the position of sensed magnet along the $y$ axis. Clearly, the standard deviation curves can correctly represent the $y$ position of the magnet, indicating the high sensing accuracy of the proposed sensing array, which can be easily recognized via the images shown in the insets.

The proposed sensing array is designed for one-dimensional (1D) magnetic field positioning; however, the sensing result can be misled by changing the direction of the magnetic field. To overcome this limitation, the sensing array can be improved by designing a multi-dimension array, which is capable of achieving $2 \mathrm{D}$ or 3D magnetic field positioning.

\section{CONCLUSION}

In summary, the design, fabrication, and experimental measurements of a magnetic field ME sensor utilizing a laminated face shear mode PMN-PT/Metglas were reported. Weak AC/DC magnetic fields, being on the order of $2 \times 10^{-8} \mathrm{Oe}$ (AC) and $2 \times 10^{-6} \mathrm{Oe}$ (DC), can be detected. A $1 \mathrm{D}$ sensor array was also proposed for sensing the position of a weak magnetic field of $2 \times 10^{-6}$ Oe, which demonstrates its great potential for weak magnetic field sensing and positioning.

\section{ACKNOWLEDGMENTS}

S. Zhang acknowledges support from the ARC (No. FT140100698).

The authors declare that they have no conflict of interest. The authors would like to thank METGLAS ${ }^{\star}$ Inc. for providing metglas sample.

\section{DATA AVAILABILITY}

The data that support the findings of this study are available on request from the corresponding author. The data are not publicly available due to privacy or ethical reasons.

\section{REFERENCES}

${ }^{1}$ R. Jahns, R. Knöchel, H. Greve, E. Woltermann, E. Lage, and E. Quandt, in IEEE International Symposium on Medical Measurements and Applications (IEEE, Bari, 2011), p. 107.

${ }^{2}$ H. Lin, M. Zaeimbashi, N. Sun, X. Liang, H. Chen, C. Dong, A. Matyushov, X. Wang, Y. Guo, Y. Gao, and N.-X. Sun, in IEEE International Microwave Biomedical Conference (IMBioC) (IEEE, Philadelphia, PA, 2018), p. 13.

${ }^{3}$ Y. J. Wang, J. Q. Gao, M. H. Li, Y. Shen, D. Hasanyan, J. F. Li, and D. Viehland, Philos. Trans. R. Soc. A 372, 20120455 (2014).

${ }^{4}$ Z. Chu, M. PourhosseiniAsl, and S. Dong, J. Phys. D Appl. Phys. 51, 243001 (2018).

${ }^{\mathbf{5}}$ A. V. Turutin, J. V. Vidal, I. V. Kubasov, A. M. Kislyuk, M. D. Malinkovich,

Y. N. Parkhomenko, S. P. Kobeleva, O. V. Pakhomov, A. L. Kholkin, and N. A. Sobolev, Appl. Phys. Lett. 112, 262906 (2018).
${ }^{6}$ M. Li, A. Matyushov, C. Dong, H. Chen, H. Lin, T. Nan, Z. Qian, M. Rinaldi, Y. Lin, and N. X. Sun, Appl. Phys. Lett. 110, 143510 (2017).

${ }^{7}$ See https://www.lakeshore.com/products/categories/overview/magnetic-products/ gaussmeters-teslameters/model-475-dsp-gaussmeter for the information of the sensitivity of a commercial gaussmeter.

${ }^{8}$ G. Liu, W. Jiang, J. Zhu, and W. Cao, Appl. Phys. Lett. 99, 162901 (2011).

${ }^{9}$ S. Zhang and T. R. Shrout, IEEE Trans. Ultrason. Eng. 57, 2138 (2010).

${ }^{10}$ Z. Chu, H. Shi, W. Shi, G. Liu, J. Wu, J. Yang, and S. Dong, Adv. Mater. 29, 1606022 (2017).

${ }^{11}$ J. Gao, D. Gray, Y. Shen, J. Li, and D. Viehland, J. Appl. Phys. 99, 153502 (2011).

${ }^{12}$ F. Li, S. Zhang, Z. Xu, X. Wei, and T. R. Shrout, Adv. Funct. Mater. 21, 2118 (2011).

${ }^{13}$ X. Jiang, J. Kim, and K. Kim, Crystals 4, 351 (2014).

${ }^{14}$ Y. Wang, P. Finkel, J. Li, and D. Viehland, J. Appl. Phys. 113, 172904 (2013).

${ }^{15}$ S. Zhang, W. Jiang, R. J. Meyer, Jr., F. Li, J. Luo, and W. Cao, J. Appl. Phys. 110, 064106 (2011)

${ }^{16}$ S. Zhang, F. Li, W. Jiang, J. Luo, R. J. Meyer, Jr., W. Cao, and T. R. Shrout, Appl. Phys. Lett. 98, 182903 (2011).

${ }^{17}$ P. Berik, W. Y. Chang, and X. Jiang, Proc. SPIE 10598, 105981F (2018).

${ }^{18}$ K. Kim, S. Zhang, and X. Jiang, IEEE Trans. Ultrason. Eng. 59, 2548 (2012).

${ }^{19} \mathrm{~S}$. Li, W. Jiang, L. Zheng, and W. Cao, Appl. Phys. Lett. 102, 183512 (2013).

${ }^{20}$ Z. Chen, X. Li, P. Ci, G. Liu, and S. Dong, Rev. Sci. Instrum. 86, 035002 (2015).

${ }^{\mathbf{2 1}}$ X. Shi, W. Huang, F. Li, Z. Li, Z. Xu, X. Jiang, and X. Wei, AIP Adv. 6, 115017 (2016).

${ }^{22}$ S. Park, M. Peddigari, G.-T. Hwang, W.-H. Yoon, A. Kumar, and J. Ryu, Appl. Phys. Lett. 115, 102901 (2019).

${ }^{23}$ J. Ryu, A. V. Carazo, K. Uchino, and H.-E. Kim, Jpn. J. Appl. Phys. 40, 4948 (2001).

${ }^{24}$ J. Ryu, S. Priya, K. Uchino, and H.-E. Kim, J. Electroceram. 8, 107 (2002).

${ }^{25}$ I. M. Daniel and O. lshai, Engineering Mechanics of Composite Materials (Oxford University Press, New York, 1994), Vol. 3.

${ }^{26}$ J. V. Vidal, A. V. Turutin, I. V. Kubasov, M. D. Malinkovich, Y. N. Parkhomenko, S. P. Kobeleva, A. L. Kholkin, and N. A. Sobolev, IEEE Trans. Ultrason. Eng. 64, 1102 (2017).

${ }^{27}$ L. Yana, Z. Xing, Z. Wang, T. Wang, G. Lei, J. Li, and D. Viehland, Appl. Phys. Lett. 94, 192902 (2009).

${ }^{28}$ C. Fang, J. Jiao, J. Ma, D. Lin, H. Xu, X. Zhao, and H. Luo, J. Phys. D Appl. Phys. 48, 465002 (2015).

${ }^{29}$ V. Annapureddy, H. Palneedi, W.-H. Yoon, D.-S. Park, J.-J. Choi, B.-D. Hahn, C.-W. Ahn, J.-W. Kim, D.-Y. Jeong, and J. Ryu, Sens. Actuators A 260, 206 (2017).

${ }^{30}$ C. W. Nan, Phys. Rev. B 50, 6082 (1994).

${ }^{31}$ C. W. Nan, M. Li, and J. H. Huang, Phys. Rev. B 63, 144415 (2001).

${ }^{32}$ C. W. Nan, G. Liu, and Y. Lin, Appl. Phys. Lett. 83, 4366 (2003).

${ }^{33}$ C. W. Nan, M. I. Bichurin, S. Dong, D. Viehland, and G. Srinivasan, J. Appl. Phys. 103, 191-293 (2008).

${ }^{34}$ D. A. Pan, Y. Bai, W. Y. Chu, and L. J. Qiao, J. Phys. Condens. Matter 20, 025203 (2008)

${ }^{35}$ G. T. Hwang, H. Palneedi, B. M. Jung, S. J. Kwon, M. Peddigari, Y. Min, J. W. Kim, C. W. Ahn, J. J. Choi, B. D. Hahn, J. H. Choi, W. H. Yoon, D. S. Park, S. B. Lee, Y. Choe, K. H. Kim, and J. Ryu, ACS Appl. Mater. Interfaces 10, 32323 (2018).

${ }^{36}$ S. Goljahi, J. Gallagher, S. J. Zhang, J. Luo, R. Sahul, W. Hackenberger, and C. S. Lynch, Smart Mater. Struct. 21, 055005 (2012).

${ }^{37}$ Y. Shen, D. Hasanyan, J. Gao, Y. Wang, J. Li, and D. Viehland, Smart Mater. Struct. 22, 095007 (2013).

${ }^{38}$ Y. Shen, J. Wang, J. Shi, S. Zhao, and J. Gao, J. Magn. Magn. Mater. 484, 164 (2019).

${ }^{39}$ Y. Shen, K. L. McLaughlin, J. Gao, D. Gray, L. Shen, Y. Wang, M. Li, D. Berry, J. Li, and D. Viehland, Smart Mater. Struct. 21, 065007 (2012).

${ }^{40}$ Z. Chu, W. Shi, H. Shi, Q. Chen, L. Wang, M. J. PourhosseiniAsl, C. Xiao, T. Xie, and S. Dong, Adv. Mater. Technol. 4, 1800484 (2018). 